\title{
Improvements in Muscle Strength Are Associated With Improvements in Walking Capacity in Young Children With Cerebral Palsy: A Secondary Analysis
}

Citation for published version (APA):

van Vulpen, L. F., de Groot, S., Rameckers, E. A. A., Becher, J. G., \& Dallmeijer, A. J. (2021). Improvements in Muscle Strength Are Associated With Improvements in Walking Capacity in Young Children With Cerebral Palsy: A Secondary Analysis. Pediatric physical therapy, 33(1), 24-30. https://doi.org/10.1097/PEP.0000000000000764

Document status and date:

Published: 01/01/2021

DOI:

10.1097/PEP.0000000000000764

Document Version:

Publisher's PDF, also known as Version of record

Document license:

Taverne

Please check the document version of this publication:

- A submitted manuscript is the version of the article upon submission and before peer-review. There can be important differences between the submitted version and the official published version of record.

People interested in the research are advised to contact the author for the final version of the publication, or visit the DOI to the publisher's website.

- The final author version and the galley proof are versions of the publication after peer review.

- The final published version features the final layout of the paper including the volume, issue and page numbers.

Link to publication

\footnotetext{
General rights rights.

- You may freely distribute the URL identifying the publication in the public portal. please follow below link for the End User Agreement:

www.umlib.nl/taverne-license

Take down policy

If you believe that this document breaches copyright please contact us at:

repository@maastrichtuniversity.nl

providing details and we will investigate your claim.
}

Copyright and moral rights for the publications made accessible in the public portal are retained by the authors and/or other copyright owners and it is a condition of accessing publications that users recognise and abide by the legal requirements associated with these

- Users may download and print one copy of any publication from the public portal for the purpose of private study or research.

- You may not further distribute the material or use it for any profit-making activity or commercial gain

If the publication is distributed under the terms of Article $25 \mathrm{fa}$ of the Dutch Copyright Act, indicated by the "Taverne" license above, 


\title{
Improvements in Muscle Strength Are Associated With Improvements in Walking Capacity in Young Children With Cerebral Palsy: A Secondary Analysis
}

\author{
Liesbeth F. van Vulpen, PT, PhD; Sonja de Groot, PhD; Eugene A. A. Rameckers, PT, PhD; Jules G. Becher, MD, PhD; \\ Annet J. Dallmeijer, PhD
}

Amsterdam Rehabilitation Research Center/Reade (Drs van Vulpen and de Groot), Amsterdam, the Netherlands; Centre for Human Movement Sciences, University Medical Center Groningen, University of Groningen (Dr de Groot), Groningen, the Netherlands; Department of Rehabilitation Medicine, School for Public Health and Primary Care (CAPHRI) (Dr Rameckers), Maastricht University, Maastricht, the Netherlands, and Rehabilitation Science, University of Hasselt (Dr Rameckers), Hasselt, Belgium, and Adelante Center of Expertise in Rehabilitation and Audiology (Dr Rameckers), Valkenburg and Hoensbroek, the Netherlands, and University for Professionals for Pediatric Physical Therapy, AVANSplus (Dr Rameckers), Breda, the Netherlands; Amsterdam Movement Sciences, Department of Rehabilitation Medicine, Amsterdam UMC, Vrije Universiteit Amsterdam (Drs Becher and Dallmeijer), Amsterdam, the Netherlands.

Purpose: To evaluate whether changes in lower-limb muscle strength explain changes in walking capacity during 14-week periods of usual care, power training and follow-up for children with spastic cerebral palsy.

Methods: Secondary analysis of a previously conducted double-baseline controlled trial of 22 children with spastic cerebral palsy. Generalized estimating equations were used to evaluate the relationships between within-subject changes in isometric muscle strength and walking capacity over 3 periods.

Results: Changes in hip abductor strength were associated with changes in the Muscle Power Sprint Test, changes in gastrocnemius and hip abductor strength were associated with changes in the Shuttle Run Test, and changes in gastrocnemius strength were associated with changes in the 1-minute walk test. All associations supported better walking capacity with increased strength.

Conclusion: Walking capacity, especially sprint capacity, can be improved by increasing strength by functional power training in this population. (Pediatr Phys Ther 2021;33:24-30)

Key words: cerebral palsy, children, muscle strength, strength training, walking

\author{
0898-5669/110/3301-0024 \\ Pediatric Physical Therapy \\ Copyright $\odot 2020$ Academy of Pediatric Physical Therapy of the American \\ Physical Therapy Association
}

Correspondence: Liesbeth F. van Vulpen, PT, PhD, Amsterdam Rehabilitation Research Center/Reade, PO Box 58271, 1040 HG Amsterdam, the Netherlands (l.v.vulpen@reade.nl).

Grant Support: This study has been funded by Mitialto Foundation, Duyvensz-Nagel Foundation, Dutch Rehabilitation Fund and Royal Dutch Society for Physical Therapy.

At the time this article was written, Liesbeth van Vulpen was a PhD student at Amsterdam Rehabilitation Research Center/Reade, Amsterdam, the Netherlands, in collaboration with the Department of Rehabilitation Medicine, VU University Medical Center, Amsterdam, the Netherlands. She was also working as a pediatric physical therapist at Reade, Center for Rehabilitation and Rheumatology, Amsterdam, the Netherlands.

Trial Registration NTR5189.

Supplemental digital content is available for this article. Direct URL citations appear in the printed text and are provided in the HTML and PDF versions of this article on the journal's Web site (www.pedpt.com).

The authors declare no conflicts of interest.

DOI: 10.1097/PEP.0000000000000764

\section{INTRODUCTION}

Children with cerebral palsy (CP) have impaired walking capacity because of their motor impairments. ${ }^{1}$ These motor impairments include spasticity, loss of selective motor control and muscle weakness. ${ }^{1}$ As a consequence these children experience problems in keeping up with their peers who are developing typically, during activities, such as playing in the schoolyard, participating in gym class, and taking part in school outings. ${ }^{2}$ Optimizing walking capacity (previously defined as sprint capacity, walking speed, and running endurance ${ }^{3}$ ) is, therefore, an important aim of treatment at a young age.

Apart from spasticity and reduced selective motor control, muscle weakness is often reported as an important factor affecting walking capacity. ${ }^{1}$ Children with $\mathrm{CP}$ who are able to walk have approximately $36 \%$ to $82 \%$ of the muscle strength of children who are developing typically., 4 Previous crosssectional studies have reported a moderate to strong relationship between leg muscle strength and walking capacity in children and young adults with $\mathrm{CP}$, showing better walking capacity in those with stronger muscles., ${ }^{4-8}$ Clinicians and researchers, therefore, focused on muscle strengthening with the aim to increase walking capacity in these children. Unfortunately, systematic reviews supported that there is inconclusive 
evidence that the walking capacity can be improved by increasing muscle strength through progressive resistance exercises. ${ }^{9}, 10$ Some studies have shown increases in muscle strength of some of the targeted leg muscles after progressive resistance training programs, but this was not accompanied by increases in walking capacity. ${ }^{11,12}$ Lack of specificity of the resistance training programs is a possible explanation for the absence of functional effects. ${ }^{13,14}$ Moreau et al ${ }^{15}$ suggested that, to get functional improvements, the strength training velocity has to be at a higher, more functional movement velocity than generally used in resistance training. Children with $\mathrm{CP}$ have a reduced capacity to rapidly generate force ${ }^{15}$ and a reduced sprinting capacity. ${ }^{16,17}$ This capacity is especially needed in playing activities such as running and sprinting games on the playground and in sports. Therefore, to increase walking capacity, a task-specific training approach with higher movement velocity in progressively loaded resistance training exercises was developed, and incorporated relevant functional activities such as walking and sprinting. ${ }^{3}$ In this functional power training study, exercises, like running and stair climbing, were conducted by children at maximal effort at $70 \%$ of their maximal speed, while the exercise was progressively loaded to ensure the principles of strength training (ie, specificity, adaptation, and overload). ${ }^{14}$

Recently, we published the results of this functional powerbased resistance training program (MegaPower training) on muscle strength and walking capacity in young children with CP. ${ }^{14}$ The results supported that functional power training was highly effective for increasing muscle strength (18\%-128\%) of the ankle plantar flexors, hip abductors, and knee extensors. In addition, sprint capacity, walking speed, and running endurance improved by $83 \%, 13 \%$, and $56 \%$, respectively. ${ }^{14}$ It is, however, not yet clear to what extent the increased muscle strength explained the improved walking capacity, and which muscle groups accounted for the improvements in walking capacity.

Therefore, the aim of this study was to evaluate whether changes in muscle strength were associated with changes in walking capacity. We performed a secondary analysis of the data of the before-mentioned functional power training trial, ${ }^{14}$ assessing to what extent changes in muscle strength were associated with changes in walking capacity. We combined data from a 14-week usual care period, a 14-week functional power training period, and a 14-week follow-up (usual care).

\section{METHODS}

\section{Participants}

Participants were children aged 4 to 10 years, with a spastic CP whose function was classified as Gross Motor Function Classification System (GMFCS) I and II. Parents and/or the children had a treatment question related to walking capacity (such as being able to walk longer or faster). Children had to be able to understand and follow simple instructions. Exclusion criteria were treatment with botulinum toxin A in the lower limb and/or serial casting of the lower limb less than 6 months before the start of the functional power training and selective dorsal rhizotomy (SDR) treatment less than 1 year before the functional power training. Three children had an SDR treatment 5 to 1.5 years before the start of the functional power training.

\section{Design and Procedure}

This study consists of a secondary analysis of data that were collected in a study on the effects of functional power training on walking capacity and leg strength in young children with $\mathrm{CP}^{14}$ The study had a double-baseline design, with 3 different periods: (1) a 14-week usual care period, (2) a 14-week functional power training period, and (3) a 14-week follow-up period with usual care (Figure 1). ${ }^{3}$

Measurements were taken on 4 occasions: pre-study (start of the 14-week usual care period), pre-intervention, postintervention, and at 14-week follow-up. During the functional power training period, children participated in loaded functional exercises like running and walking with a progressive load, 3 times a week for 60 minutes. Exercises were performed at $50 \%$ to $70 \%$ of their maximum unloaded speed by applying resistance to the movement with an external load. Each exercise was performed for 25 seconds on maximal effort, with a resting period of 30 to 50 seconds, and 6 to 8 repetitions for each exercise. ${ }^{3}$ The activity and rest ratio had similarities to that of high-intensity interval training, ${ }^{18}$ which previously has shown to increase both sprint and endurance in children with CP. ${ }^{19}$ Children followed their regular physical therapy sessions during usual care and follow-up periods. Detailed description of the functional power training study has been previously published. ${ }^{3}$

For descriptive purposes, GMFCS and type of CP were determined by a pediatric physical therapist together with a physiatrist. ${ }^{20}$ To determine the more and less affected leg of each child, selective motor control of knee extension and ankle dorsiflexion were tested by the modified Trost Selective Motor Control test. ${ }^{21}$

Medical Ethics Committee of the Slotervaart Medical Center and Reade rehabilitation Research Center in Amsterdam, the Netherlands, approved this study, and written informed consent was obtained from the parents of each participant.

\section{Setting}

Participants were recruited from a rehabilitation center, 2 special needs schools for children with physical disabilities and an outpatient clinic of an university medical center (see Supplemental Digital Content 1, available at: http://links.lww.com/ PPT/A307).

\section{Measurements}

Walking Capacity. Walking capacity was defined as sprint capacity, endurance, and walking speed. ${ }^{3}$ Sprint capacity of the child was expressed in mean power and peak power, measured with the $6 \times 15-\mathrm{m}$ Muscle Power Sprint Test (MPST). ${ }^{3,22}$ The power output for each of the 6 sprint runs was estimated using

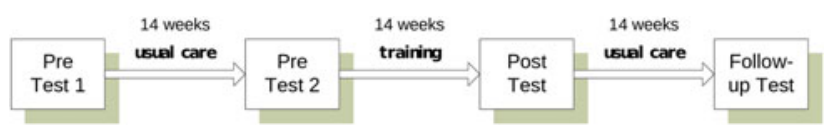

Fig. 1. Double-baseline research protocol with follow-up. Reprinted with permission from van Vulpen et al ${ }^{3}$ ¿ 2017 Wolters Kluwer Health Inc, and Academy of Pediatric Physical Therapy of the American Physical Therapy Association. This figure is available in color online (www.pedpt.com). 
sprinting velocity and body mass with the following equations: Power $(\mathrm{W})=$ Force $\times$ Velocity, where velocity is calculated from time to complete 15-m sprint, and force is calculated as body mass $\times$ acceleration. ${ }^{22}$ Mean power was defined as the average power output of the 6 runs. The reliability of the MPST, with an intraclass correlation coefficient (ICC) of 0.97 , and the feasibility and construct validity are good. ${ }^{22}$ Running endurance was measured with the 10-m Shuttle Run Test (SRT), modified for children with CP GMFCS levels I and II. ${ }^{23}$ Reliability and validity of the SRT are good with an ICC of 0.87 to $0.99 .{ }^{23}$ Walking speed was measured with the distance covered in the 1-minute walk test (1MWT), with instructions to walk as fast as possible in 1 minute. ${ }^{24}$ The reliability (ICC $=0.94$ ) and validity of the $1 \mathrm{MWT}$ are good. ${ }^{24}$

Muscle Strength. Isometric muscle strength in both legs of plantar flexors with knees extended (m. gastrocnemius [GASTR]) and with knees flexed (m. soleus [SOL]), knee extensors (KE), and hip abductors (HA) was measured with a hand-held dynamometer (microFET, Biometrics BV, Almere, the Netherlands) using the make method where the child gradually builds up force against the dynamometer for about 5 seconds. ${ }^{25}$ A standardized protocol was used for positioning of the child, joint fixation, and joint positioning. ${ }^{25}$ The lever arm was measured as the distance from the axis of rotation to hand held dynamometer placement, as described in a study by van Vulpen et al. ${ }^{25}$ Torque (Nm) was calculated by multiplying force (Newton) by the length (meter) of the lever arm. Each strength test was performed 3 times and maximum force for each repetition was registered. To improve reliability, strength tests were measured at 2 different test days. The mean of the 6 measurements ( 2 test days with 3 repetitions on each day) per muscle group was used in the analysis. ${ }^{25}$ Isometric strength measurements have good reliability in children with $\mathrm{CP}$ when measured with 3 repetitions in 2 different test occasions (ICC $=0.88$ 0.98). ${ }^{25}$

Dynamic muscle strength of plantar flexors of the more and less affected leg was measured with a standing heel-rise test on 1 leg following a standardized protocol. ${ }^{25}$ The maximum number of repetitions for each leg was noted. The test stopped when the child leaned or pushed down on the examiner, the child's knees flexed, or when the child gave up despite encouragement. Moderate to good reliability is found for the heel-rise test in children with CP (ICC $=0.86-0.98){ }^{25}$

See Supplemental Digital Content 2 (available at: http:// links.lww.com/PPT/A308).

\section{Statistical Analysis}

Changes in muscle strength and walking capacity during the usual care, functional power training, and follow-up periods were calculated and expressed as a percentage of the pretest of each period, except for the dynamic muscle strength (heelrise test), which was expressed as change in number of repetitions possible. Generalized estimating equation (GEE) analysis was used to evaluate the relationship between the changes in muscle strength (independent variable) and changes in walking capacity (dependent variable). For each subject, data over 3 measurement periods (usual care, intervention, and follow-up) were included in the models. GEE takes the dependency of these related samples (repeated measurements due to the 3 periods, and more and less affected leg) into account.

Univariable modeling was applied for each walking capacity outcome, and each strength parameter including changes in walking capacity and strength over 3 measurements periods (usual care, intervention, and follow-up). Multivariable modeling was used with a backward selection procedure with the changes in isometric muscle strength of all muscles (GASTR, SOL, HA, and KE) of both legs included as independent variables. The multivariable models were completed for each walking capacity outcome separately. Independent variables (changes in muscle strength) were removed from the model, starting with the least significant independent variable, until only variables remained that met the criteria $P<.05$. All statistical analyses were performed using SPSS Statistics 22.0 software (IBM Corporation, New York).

\section{RESULTS}

\section{Characteristics of the Study Population}

Characteristics of the 22 participants in this study are in Table 1. One participant was hospitalized during the follow-up measurement because of a medical problem that was unrelated to the training. There were 2 missing values for the 1MWT: 1 participant could not be tempted to walk instead of run during the posttest, and 1 participant changed footwear. One participant could not perform the isometric GASTR and SOL tests of the more affected limb because of motor control problems. Consequently, 57 to 65 data points in 22 participants were used in the univariable and multivariable regression analyses.

\section{TABLE 1}

Characteristics of Participants

\begin{tabular}{lc}
\hline Boys, $\mathrm{n}$ & 11 \\
Girls, $\mathrm{n}$ & 11 \\
Age, $\mathrm{y}$ & \\
$\quad$ Mean (SD) & $7.5(1.8)$ \\
$\quad$ Range & $4.0-10.2$ \\
Body mass, kg & \\
$\quad$ Mean (SD) & $26.0(8.2)$ \\
$\quad$ Range & $14.0-45.5$ \\
Height, $\mathrm{m}$ & \\
$\quad$ Mean (SD) & $1.24(0.11)$ \\
Range & $0.99-1.45$ \\
GMFCS I, n & 10 \\
GMFCS II, $\mathrm{n}$ & 12 \\
Unilateral spastic CPa ${ }^{\mathrm{a}}, \mathrm{n}$ & 9 \\
Bilateral spastic CP, $\mathrm{n}$ & 13 \\
Regular school, $\mathrm{n}$ & 13 \\
Special needs education, $\mathrm{n}$ & 9 \\
AFO ${ }^{\mathrm{b}}$ in daily life, $\mathrm{n}$ & 13 \\
No AFO in daily life, $\mathrm{n}$ & 9 \\
\hline
\end{tabular}

Abbreviations: AFO, ankle-foot orthosis; CP, cerebral palsy; GMFCS, Gross Motor Function Classification System; SCPE, Surveillance of Cerebral Palsy in Europe.

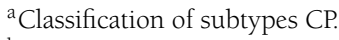

${ }^{b}$ On 1 limb or both. Supramalleolar orthosis and orthopedics shoes or registered as no $\mathrm{AFO}$ in daily life. 
TABLE 2

Changes in Walking Capacity, Shuttle Run Test, 1-Minute Walk Test, Muscle Strength ${ }^{a}$

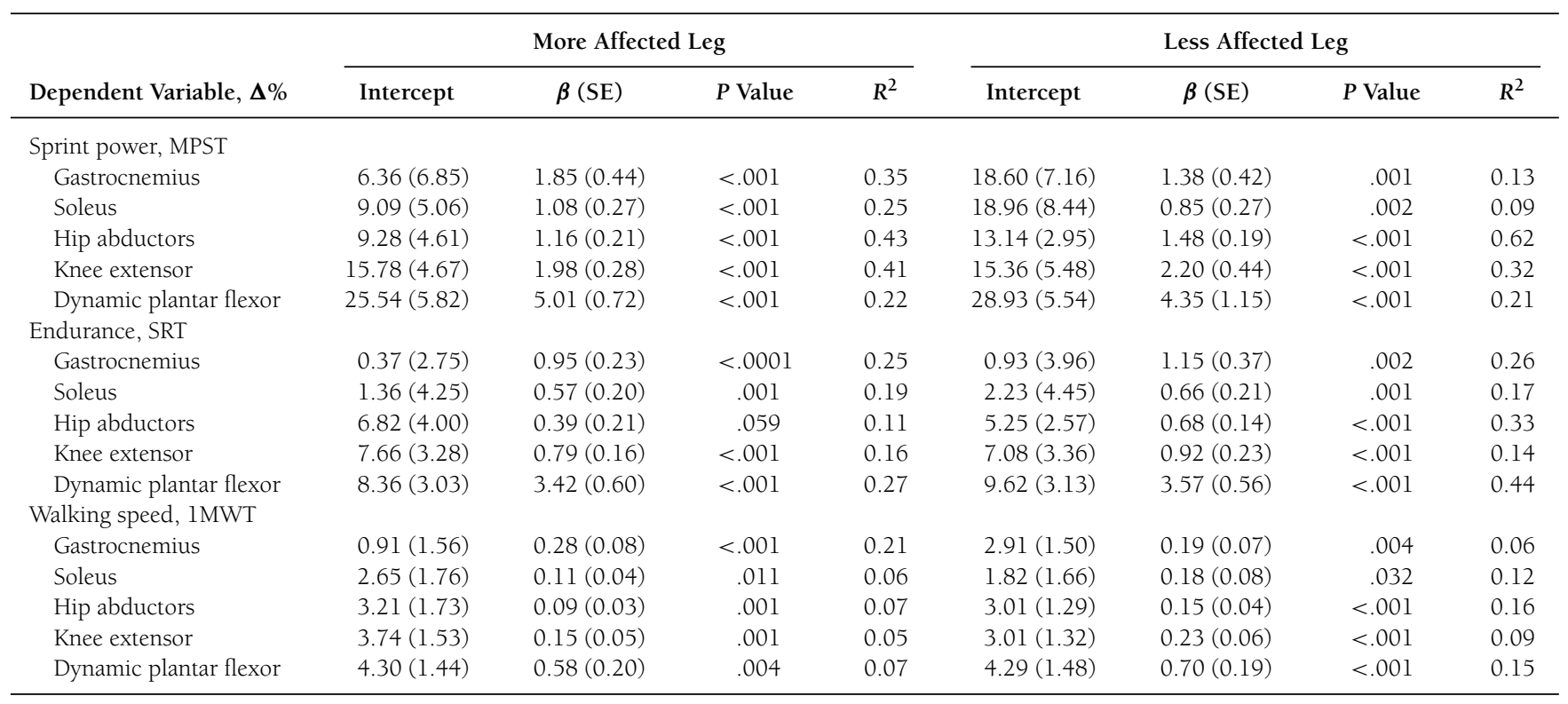

Abbreviations: MPST, Muscle Power Sprint Test; 1MWT, 1-minute walk test; SE, standard error; SRT, Shuttle Run Test.

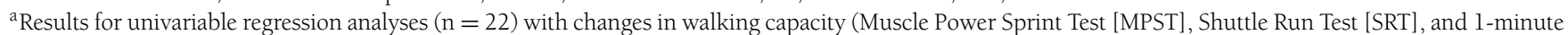

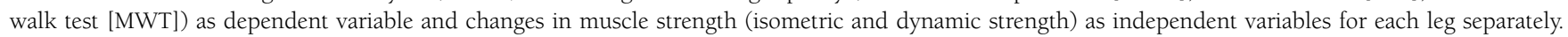

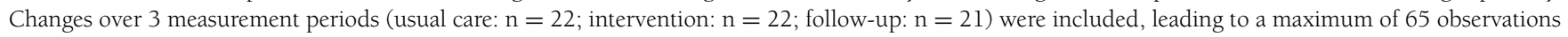
in each model.

\section{Univariable Regression}

Table 2 has the results for univariable regression analyses with relationships between changes in walking capacity and changes in muscle strength. All muscle groups supported significant associations with the MPST ( $R^{2}$ ranging from 0.09 to $0.62)$, SRT ( $R^{2}$ ranging from 0.11 to 0.44$)$, and 1MWT $\left(R^{2}\right.$ ranging from 0.05 to 0.21 ). The regression coefficient $(\beta)$ indicates, for example, that for every increase of $10 \%$ in gastrocnemius strength of the more affected leg a mean increase of $18.5 \%$ in sprint capacity was found. The relation between changes in walking capacity and changes in GASTR of the more affected leg and the less affected leg is visualized in Figure 2.

\section{Multivariable Regression}

Table 3 has the results of multivariable regression. Changes in HA strength of both legs had a significant association $\left(R^{2}=\right.$ $0.66)$ with MPST changes. Changes in GASTR and HA strength, both in the less affected leg, had a significant association $\left(R^{2}\right.$ $=0.43)$ with SRT changes. Changes in GASTR strength in the more affected leg had a significant association $\left(R^{2}=0.21\right)$ with 1MWT changes. KE and SOL strength changes had no significant associations with walking capacity changes in the multivariable models.

See Supplemental Digital Content 3 (available at: http:// links.lww.com/PPT/A309) for more details.

\section{DISCUSSION}

The results of the present study indicated that larger relative changes in muscle strength are associated with larger rela- tive changes in walking capacity. Especially the improvements in hip abductor (HA) and gastrocnemius (GASTR) muscle strength were associated with better sprint capacity $\left(R^{2}=0.66\right)$, walking endurance $\left(R^{2}=0.43\right)$, and to a lesser extent to walking speed $\left(R^{2}=0.21\right)$. Our findings suggest that the increased walking capacity after functional power training is mainly due to the increased strength of HA and GASTR. Largest improvements were found in sprint capacity, which was in line with the content (loaded sprint exercises) of the functional power training intervention.

We previously described the positive effects of the functional power training on muscle strength and walking capacity at group level using the same data as in the current study. ${ }^{14}$ The participating children acted as their own controls in this doublebaseline design: a 14-week usual care period was compared with a 14-week functional power training intervention that followed immediately after the usual care period. ${ }^{14}$ No significant changes in muscle strength and walking capacity were seen in the usual care period. Large increases in strength and walking capacity were found in the functional power period, and subsequently in the follow-up period muscle strength remained stable while only small decreases in walking capacity were found. ${ }^{14}$ In the current study, we supported that these relative within-subject muscle strength changes (no changes, large increases, and small decreases) over these 3 periods could explain the relative changes in walking capacity to a large extent.

Results of a systematic review and meta-analysis supported that improvements in walking capacity are not expected after resistance training; however, these results were based on studies using strength rather than power-based, resistance training dose. ${ }^{9,10}$ In contrast, the strong associations between changes in muscle strength and changes in walking capacity in the 

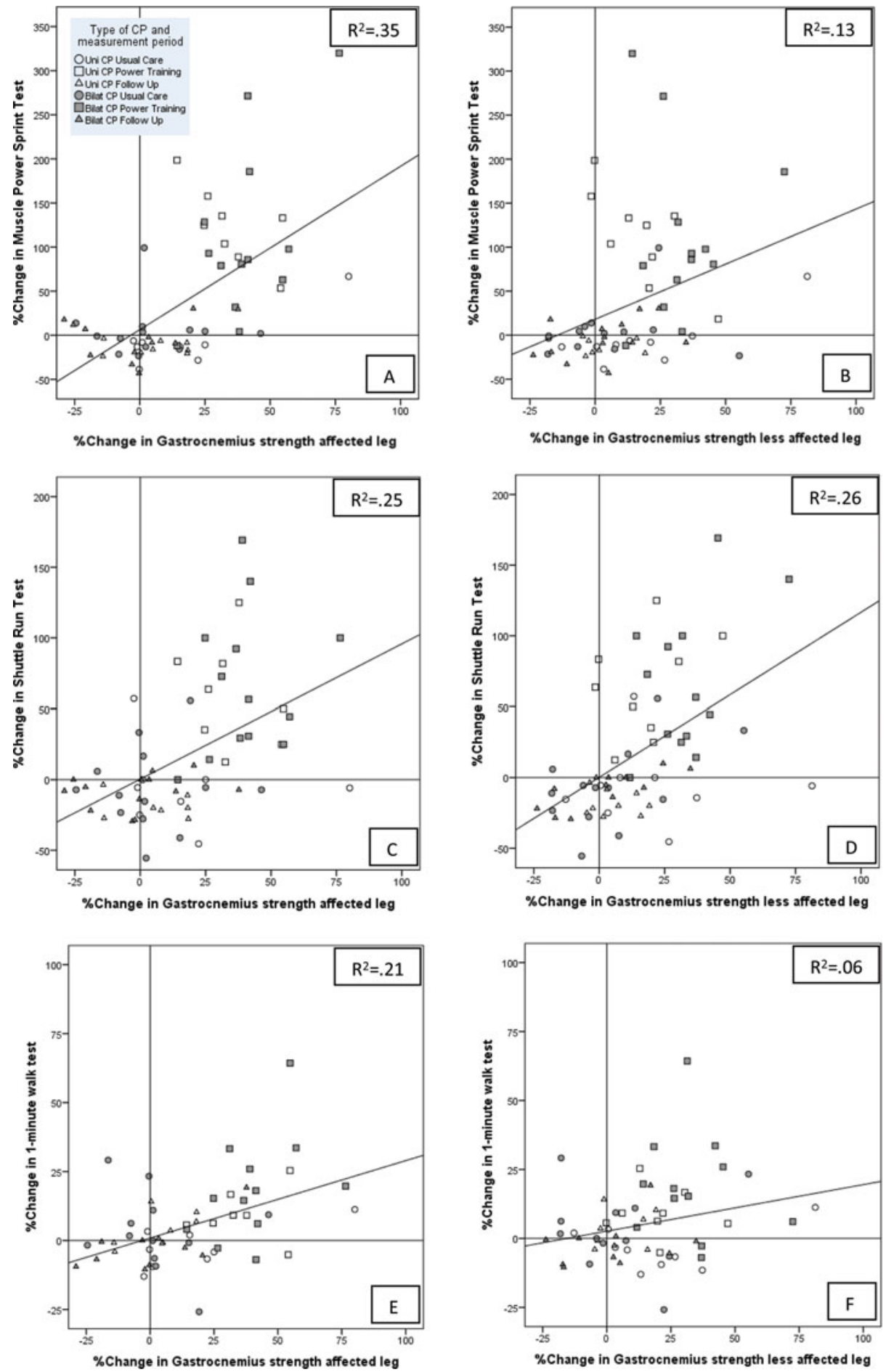

Fig. 2. Changes in walking capacity (Muscle Power Sprint Test, Shuttle Run Test, and 1-minute walk test) with changes in isometric muscle strength of m. gastrocnemius (plantar flexor knees extended more and less affected leg) over 3 periods (14 weeks' usual care, 14 weeks' power training, and 14 weeks' follow-up with usual care). This figure is available in color online (www.pedpt.com).

present study indicate that improving strength may improve walking capacity. This discrepancy might be attributed to the character of the power-based resistance training and secondly to the magnitude of the increases found after the functional power training. The specificity of the functional power training could be the explanation for these strong associations due to the higher movement velocity used in the progressive loaded powerbased resistance training, and the incorporation of functional movements like walking and sprinting. The larger increases in muscle strength (18\%-128\%), compared with previous resistance training studies, ${ }^{11,12}$ may explain its positive effect on walking capacity.

It is suggested that muscle strength increases in children are primarily due to neuromuscular adaptations instead of muscle volume changes. ${ }^{26}$ Rose and McGill ${ }^{27}$ examined the neuromuscular activation and motor unit firing characteristics in children 


\section{TABLE 3}

Relationship Between Changes in Walking Capacity and Changes in Isometric ${ }^{a}$

\begin{tabular}{|c|c|c|c|c|}
\hline $\begin{array}{l}\text { Dependent Variables Walking } \\
\text { Capacity }\end{array}$ & $\begin{array}{l}\text { Independent Variable in Final Model } \\
\text { Isometric Muscle Strength }\end{array}$ & $\beta(\mathrm{SE})$ & $P$ Value & $R^{2}$ Final Model \\
\hline \multirow[t]{2}{*}{ Muscle Power Sprint Test, $\Delta \%$} & Intercept & $7.13(3.41)$ & & \\
\hline & Hip abductors less affected leg $(\Delta \%)$ & $1.17(0.21)$ & $<.001$ & 0.66 \\
\hline \multirow[t]{3}{*}{ Shuttle Run Test, $\Delta \%$} & Intercept & $-1.08(3.83)$ & & \\
\hline & Gastrocnemius more affected leg $(\Delta \%)$ & $-0.15(0.22)$ & .508 & \\
\hline & Hip abductors less affected leg $(\Delta \%)$ & $0.67(0.19)$ & $<.001$ & 0.43 \\
\hline \multirow[t]{3}{*}{ 1-min walk test, $\Delta \%$} & Intercept & $1.04(1.59)$ & & \\
\hline & Gastrocnemius more affected leg $(\Delta \%)$ & $0.30(0.11)$ & .005 & \\
\hline & Gastrocnemius less affected leg $(\Delta \%)$ & $-0.03(0.11)$ & .801 & 0.21 \\
\hline
\end{tabular}

Abbreviation: SE, standard error.

${ }^{a}$ Results of multiple regression analyses $(n=22)$ for the relationship between changes in walking capacity (dependent variable) and changes in isometric strength (independent variable) for both legs. Changes over 3 measurement periods (usual care: $\mathrm{n}=22$; intervention: $\mathrm{n}=22$; follow-up: $\mathrm{n}=21$ ) were included, leading to a maximum of 65 observations in each model.

with CP. Weakness in the GASTR appears to result, in part, from the inability of the damaged pathways to provide sufficient excitatory drive to the motor neuron pool to fully activate all available motor units. ${ }^{27}$ The authors ${ }^{27}$ suggested that treatments aimed at building strength, such as high-intensity strength training, may be effective for reducing the movement deficit in gross motor functioning known in children with $\mathrm{CP}$ and improving gait in that they increase voluntary excitatory drive and muscle activation. This supports the idea that neuromuscular adaptations increase walking capacity rather than muscle volume changes alone. Our results supported that there is an association between strength and sprint capacity; however, it is unknown what the cause and effect is. Improvements in HA and GASTR strength might lead to a better sprint capacity, but it can also be the other way around. This might be an interesting subject for future research.

The importance of the HA and GASTR muscle strength for improvements in walking capacity is in agreement with previous studies assessing multivariable cross-sectional associations between strength of similar leg muscles and walking in children with $\mathrm{CP}^{4,6-8,28}$ The difference between these cross-sectional studies and our study was that we evaluated the association in relative changes between strength and walking capacity over 3 periods (ie, longitudinally). We used percent change rather than absolute values because of the heterogeneity of the group. For example, child 1 supported an increase of $125 \%$ in sprint power (4 W pre-intervention to $9 \mathrm{~W}$ post-intervention) and child 2 supported an increase of $8 \%$ ( $60 \mathrm{~W}$ pre-intervention to $65 \mathrm{~W}$ post-intervention). If we would have used absolute values in this example, we would see an increase of $5 \mathrm{~W}$ in both children. However, when taking the percentage of change then we are taking into account that child 1 changed much more in sprint power than child 2 . Taken relative changes in our analysis could potentially have strengthen the results.

Lack of an association between changes in KE and changes in walking capacity in the multivariable models in our study was in agreement with the findings of a study of van der Krogt et al. ${ }^{29}$ van der Krogt et al ${ }^{29}$ developed muscle-driven simulations of normal walking and then progressively weakened all major muscle groups to evaluate how muscle weakness could be tolerated before execution of normal gait became impossible. They found that gait appears most robust to weakness of hip and knee extensors, which can tolerate weakness well and without a substantial increase in total muscle cost. ${ }^{29}$ However, gait was most sensitive to weakness of plantar flexors, hip abductors, and hip flexors, since weakness in these muscle could be tolerated only to a limited extent, and resulted in a large increase in total muscle cost. ${ }^{29}$ This might indicate that gait training should concentrate more on improving HA and GASTR strength and the strengthening exercises should be incorporated in walking and running activities. However, Moreau et $\mathrm{al}^{13}$ found increases in the 1MWT after power training on a Biodex dynamometer of the KE in adolescents with $\mathrm{CP}$, indicating that power changes in other muscle groups may also contribute to better walking capacity. It remains to be investigated to what extent strength changes in muscle groups that were not measured in our study, such as hip extensors and flexors and the knee flexors, contribute to the changes in walking capacity. A limitation of this study is that we did not measure muscle power production of each muscle group. It is unknown whether the changes in isometric and dynamic strength measured in our study were accompanied with changes in muscle power production.

The functional power training focused on increasing plantar flexor strength by using exercises that aimed to elicit a powerful push-off during walking and running. ${ }^{3}$ Plantar flexors are also often targeted in treatments with botulinum toxin injections, orthoses, and serial casting to reduce spasticity, to increase range of motion in the ankle joint, and to increase walking quality. However, as a side effect, these treatments may exacerbate muscle weakness. ${ }^{30}$ The present results suggest that functional power training increases isometric- and dynamic plantar flexor strength and walking capacity and it therewith may be a useful intervention to ameliorate the muscle weakness associated with botulinum toxin injections and serial casting.

In our study we found that changes in muscle strength of the more and less affected leg both influenced walking capacity. Most previous cross-sectional studies, which evaluated associations between walking capacity and muscle strength in $\mathrm{CP}$, only 
included muscle strength of the nondominant, presumably the more affected, leg because of the assumption that increasing muscle strength of the nondominant side would have more effect on walking capacity than the dominant (ie, stronger) leg. ${ }^{4,6-8}$ However, a previous study by de Groot et al ${ }^{31}$ supported that strength of the less affected leg of adults with CP supported stronger correlations with cycling performance than the more affected leg. Results of our study suggest that changes in muscle strength of the more affected leg as well as the less affected leg are associated with changes in walking capacity.

\section{CONCLUSION}

Improvements in HA and GASTR muscle strength of both less and more affected legs were associated with better walking capacity in young children with $\mathrm{CP}$, suggesting that walking capacity, especially sprint capacity, can be improved by increasing strength of these muscles in this population when trained with a functional power training program. Based on our results, we can advise to add functional power training to the treatment program of young children with $\mathrm{CP}$ to increase muscle strength and walking capacity.

\section{ACKNOWLEDGMENTS}

The authors wish to thank all children, parents, and trainers who participated in this study.

\section{REFERENCES}

1. Graham HK, Rosenbaum P, Paneth N, et al. Cerebral palsy. Nat Rev Dis Primers. 2016;2:15082.

2. Bax MC, Flodmark O, Tydeman C. Definition and classification of cerebral palsy. From syndrome toward disease. Dev Med Child Neurol Suppl. 2007;109:39-41.

3. van Vulpen LF, de Groot S, Rameckers EAA, Becher JG, Dallmeijer AJ. Effectiveness of functional power training on walking ability in young children with cerebral palsy: study protocol of a double-baseline trial. Pediatr Phys Ther. 2017;29(3):275-282.

4. Dallmeijer AJ, Rameckers EA, Houdijk H, de Groot S, Scholtes VA, Becher JG. Isometric muscle strength and mobility capacity in children with cerebral palsy. Disabil Rehabil. 2017;39(2):135-142.

5. Wiley ME, Damiano DL. Lower-extremity strength profiles in spastic cerebral palsy. Dev Med Child Neurol. 1998;40(2):100-107.

6. Eek MN, Beckung E. Walking ability is related to muscle strength in children with cerebral palsy. Gait Posture. 2008;28(3):366-371.

7. Ferland C, Lepage C, Moffet H, Maltais DB. Relationships between lower limb muscle strength and locomotor capacity in children and adolescents with cerebral palsy who walk independently. Phys Occup Ther Pediatr. 2012;32(3):320-332.

8. Ross SA, Engsberg JR. Relationships between spasticity, strength, gait, and the GMFM-66 in persons with spastic diplegia cerebral palsy. Arch Phys Med Rehabil. 2007;88(9):1114-1120.

9. Franki I, Desloovere K, De CJ, et al. The evidence-base for conceptual approaches and additional therapies targeting lower limb function in children with cerebral palsy: a systematic review using the ICF as a framework. J Rehabil Med. 2012;44(5):396-405.

10. Park EY, Kim WH. Meta-analysis of the effect of strengthening interventions in individuals with cerebral palsy. Res Dev Disabil. 2014;35(2):239-249.

11. Scholtes VA, Becher JG, Janssen-Potten YJ, Dekkers H, Smallenbroek L, Dallmeijer AJ. Effectiveness of functional progressive resistance exercise training on walking ability in children with cerebral palsy: a randomized controlled trial. Res Dev Disabil. 2012;33(1):181-188.

12. Taylor NF, Dodd KJ, Baker RJ, Willoughby K, Thomason P, Graham HK. Progressive resistance training and mobility-related function in young people with cerebral palsy: a randomized controlled trial. Dev Med Child Neurol. 2013;55(9):806-812.

13. Moreau NG, Holthaus K, Marlow N. Differential adaptations of muscle architecture to high-velocity versus traditional strength training in cerebral palsy. Neurorehabil Neural Repair. 2013;27(4):325-334.

14. van Vulpen LF, de Groot S, Rameckers E, Becher JG, Dallmeijer AJ. Improved walking capacity and muscle strength after functional powertraining in young children with cerebral palsy. Neurorehabil Neural Repair. 2017;31(9):827-841

15. Moreau NG, Falvo MJ, Damiano DL. Rapid force generation is impaired in cerebral palsy and is related to decreased muscle size and functional mobility. Gait Posture. 2012;35(1):154-158.

16. Verschuren O, Maltais DB, Riet Douma-van, Kruitwagen C, Ketelaar M. Anaerobic performance in children with cerebral palsy compared to children with typically development. Pediatric Phys Ther. 2013;25(4):409-413.

17. Balemans AC, Van Wely L, De Heer SJ, et al. Maximal aerobic and anaerobic exercise responses in children with cerebral palsy. Med Sci Sports Exerc. 2013;45(3):561-568.

18. Laursen PB, Jenkins DG. The scientific basis for high-intensity interval training: optimising training programmes and maximising performance in highly trained endurance athletes. Sports Med. 2002;32(1):53-73.

19. Verschuren O, Ketelaar M, Gorter JW, Helders PJ, Uiterwaal CS, Takken T. Exercise training program in children and adolescents with cerebral palsy: a randomized controlled trial. Arch Pediatr Adolesc Med. 2007;161(11):1075-1081.

20. Bax M, Goldstein M, Rosenbaum P, et al. Executive committee for the definition of cerebral palsy. Dev Med Child Neurol. 2005;47(8):571-576.

21. Smits DW, van Groenestijn AC, Ketelaar M, Scholtes VA, Becher JG, Gorter JW. Selective motor control of the lower extremities in children with cerebral palsy: inter-rater reliability of 2 tests. Dev Neurorehabil. 2010;13(4):258-265.

22. Verschuren O, Takken T, Ketelaar M, Gorter JW, Helders PJ. Reliability for running tests for measuring agility and anaerobic muscle power in children and adolescents with cerebral palsy. Pediatr Phys Ther. 2007;19(2):108-115.

23. Verschuren O, Takken T, Ketelaar M, Gorter JW, Helders PJ. Reliability and validity of data for 2 newly developed shuttle run tests in children with cerebral palsy. Phys Ther. 2006;86(8):1107-1117.

24. McDowell BC, Humphreys L, Kerr C, Stevenson M. Test-retest reliability of a 1-min walk test in children with bilateral spastic cerebral palsy (BSCP). Gait Posture. 2009;29(2):267-269.

25. van Vulpen LF, De GS, Becher JG, de Wolf GS, Dallmeijer AJ. Feasibility and test-retest reliability of measuring lowerlimb strength in young children with cerebral palsy. Eur J Phys Rehabil Med. 2013;49(6):803-813.

26. Faigenbaum AD, Kraemer WJ, Blimkie CJ, et al. Youth resistance training: updated position statement paper from the national strength and conditioning association. J Strength Cond Res. 2009;23(5 suppl): S60-S79.

27. Rose J, McGill KC. Neuromuscular activation and motor-unit firing characteristics in cerebral palsy. Dev Med Child Neurol. 2005;47(5):329336.

28. Davids JR, Oeffinger DJ, Bagley AM, Sison-Williamson M, Gorton G. Relationship of strength, weight, age, and function in ambulatory children with cerebral palsy. J Pediatr Orthop. 2015;35(5):523-529.

29. van der Krogt MM, Delp SL, Schwartz MH. How robust is human gait to muscle weakness? Gait Posture. 2012;36(1):113-119.

30. Barber LA, Barrett RS, Gillett JG, Cresswell AG, Lichtwark GA. Neuromechanical properties of the triceps surae in young and older adults. Exp Gerontol. 2013;48(11):1147-1155.

31. de Groot S, Dallmeijer AJ, Bessems PJ, Lamberts ML, van der Woude LH, Janssen TW. Comparison of muscle strength, sprint power and aerobic capacity in adults with and without cerebral palsy. J Rehabil Med. 2012;44(11):932-938. 